\title{
Nature and physical properties of gas-mass selected galaxies using integral field spectroscopy
}

\author{
Leindert A. Boogaard (1) \\ Leiden Observatory, Leiden University, PO Box 9513, NL-2300 RA Leiden, The Netherlands \\ email: boogaard@strw.leidenuniv.nl
}

\begin{abstract}
Mapping the molecular gas content of the universe is key to our understanding of the build-up of galaxies over cosmic time. Spectral line scans in deep fields, such as the Hubble Ultra Deep Field (HUDF), provide a unique view on the cold gas content out to high redshift. By conducting 'spectroscopy-of-everything', these flux-limited observations are sensitive to the molecular gas in galaxies without preselection, revealing the cold gas content of galaxies that would not be selected in traditional studies.

In order to capitalize on the molecular gas observations, knowledge about the physical conditions of the galaxies detected in molecular gas, such as their interstellar medium conditions, is key. Fortunately, deep surveys with integral-field spectrographs are providing an unprecedented view of the galaxy population, providing redshifts and measurements of restframe UV/optical lines for thousands of galaxies.

We present the results from the synergy between the ALMA Spectroscopic Survey of the HUDF (ASPECS), with deep integral field spectroscopy from the MUSE HUDF survey and multi-wavelength data. We discuss the nature of the galaxies detected in molecular gas without preselection and their physical properties, such as star formation rate and metallicity. We show how the combination of ALMA and MUSE integral field spectroscopy can constrain the physical properties in galaxies located around the main sequence during the peak of galaxy formation.
\end{abstract}

Keywords. galaxies: high-redshift, galaxies: formation, galaxies: ISM, techniques: spectroscopic

\section{Introduction}

Recent years have seen tremendous advances in the characterization of the cosmic history of star formation and it has now been established that the star formation rate (SFR) density increased with cosmic time up to a peak at $z \sim 1-3$ and then decreased until the present (for an overview, see Madau \& Dickinson 2014). At each epoch, more massive star-forming galaxies are observed to have a higher star formation rate, establishing what has become known as the 'galaxy main sequence' (MS; Brinchmann et al. 2004; Noeske et al. 2007; Whitaker et al. 2014; Schreiber et al. 2015; Boogaard et al. 2018).

A key ingredient in our understanding of galaxy formation is the observation of the cold interstellar medium (ISM) - the 'fuel for star formation' - typically traced through carbon monoxide $\left({ }^{12} \mathrm{CO}\right.$, hereafter $\mathrm{CO}$ ) or dust continuum emission (for a review, see Carilli \& Walter 2013). Recent years have seen a significant progress in observations of $\mathrm{CO}$ at $z>1$, through targeted observations of star-forming galaxies on and above the MS (e.g., Daddi et al. 2015; Tacconi et al. 2018; Silverman et al. 2018). This has been largely driven by the incredible sensitivity of the Atacama Large Millimeter Array (ALMA), which is now revolutionizing the field of high-redshift ISM line observations, as shown by several publications in this volume. 
In order to conduct a 'complete' census of the cosmic molecular gas density, one has to probe a well defined cosmic volume without any target preselection. So called 'spectral scan surveys' are designed to do exactly that, by conducting a mosaic of observations on the sky while simultaneously scanning a large bandwidth in frequency for emission lines from the cold ISM. These are now providing the first ever constraints on the cosmic molecular gas content and hence the cosmic star formation efficiency, directly from CO observations (Decarli et al. 2014; Walter et al. 2014, 2016; Pavesi et al. 2018; Riechers et al. 2019; Decarli et al. 2019).

As molecular line emission can be elusive, having a large number of spectroscopic redshifts over the target field can provide essential redshift information to push spectral scans to their sensitivity limit. The Multi-Unit Spectroscopic Explorer (MUSE) on the Very Large Telescope (Bacon et al. 2010) is a key instrument in this context, providing 'integral field spectroscopy' over a large $1^{\prime} \times 1^{\prime}$ field-of-view from $4750-9300 \AA$ at a high observing efficiency.

Here, we will present the first results from the ALMA Spectroscopic Survey (ASPECS) in the Hubble Ultra Deep Field (HUDF) Large Program (Decarli et al. 2019), focusing specifically on the synergy between ALMA and MUSE (Boogaard et al. 2019). These results are part of a larger series of papers on the Band 3 data from ASPECS, further discussed in González-López et al. (2019); Popping et al. (2019); Aravena et al. (2019).

\section{Observations: ALMA and MUSE observations in the HUDF}

The ALMA Spectroscopic Survey consists of a mosaic of spectral scan observations targeting the deepest region of the HUDF, covering the complete ALMA Band 3 (84 $115 \mathrm{GHz})$ and Band $6(212-272 \mathrm{GHz})$ windows. These flux-limited observations allow one to detect both line emission from the ISM (mainly CO, [CI] and [CII]) as well as dust continuum at different redshifts, without any preselection of targets (see the top panels of Fig. 1). The HUDF is an excellent target for ASPECS, due to the wealth of multiwavelength data available all the from the X-ray to radio. In particular, deep $H S T$ and Spitzer photometry are key to identify the host galaxies of the molecular line emission, as the stellar light from these sources is often significantly attenuated.

The MUSE data over the HUDF provides optical spectroscopy for all galaxies in the field and has revolutionized the amount of available redshift information (increasing the number of known spectroscopic redshifts by a factor $\times 10$; Bacon et al. 2017; Inami et al. 2017). Fig. 1 shows how the galaxies at different redshifts are identified by distinct spectral features that fall within the spectrograph.

Fig. 1 also illustrates how the integral field data from ALMA and MUSE can work together: On the one hand, MUSE can provide redshift information for the galaxies detected in $\mathrm{CO}$ emission as well as physical properties from the rest-frame UV/optical spectra (depending on the redshift). On the other hand, one can leverage the large number of MUSE redshifts to search specifically for lines in the ALMA data, as well as conduct stacking in order to detect fainter sources.

\section{Results: The physical properties of the ASPECS galaxies}

We search the complete ASPECS Band 3 cube for emission lines using a matched filtering approach (González-López et al. 2019). This reveals 16 emission line candidates at high significance (very low probability of being spurious), all of which show a counterpart in the HST imaging. Using the MUSE and multi-wavelength data, we identify all as rotational transitions of $\mathrm{CO}$, with redshifts between $z=1.0-3.6\left(J_{\text {up }}=2-4\right)$. In addition, using the MUSE redshifts as prior information, we recover two additional CO lines at signal-to-noise $>3$, bringing the total sample to 18 . We convert the observed CO 


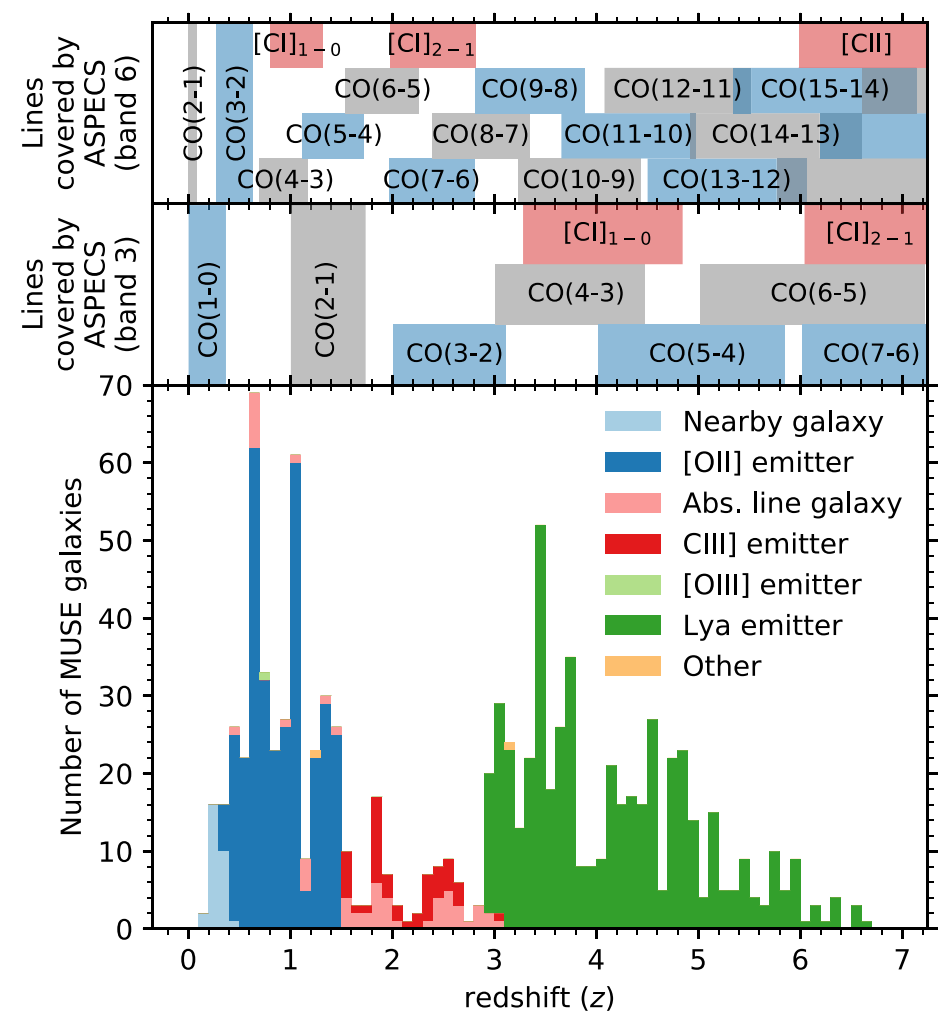

Figure 1. Top panel: Primary emission lines observable at different redshifts with the ALMA Spectroscopic Survey (ASPECS) in Band 3 and 6. Bottom panel: Histogram of all galaxies from the MUSE HUDF Survey (Bacon et al. 2017; Inami et al. 2017) that fall within the ASPECS field (Decarli et al. 2019), colored by their spectral classification. At each distinct redshift, MUSE and ALMA probe different parts of the galaxy spectra. Figure adapted from Boogaard et al. (2019).

luminosity to $\mathrm{CO}(1-0)$ using the excitation corrections derived for $z \sim 1.5$ star-forming galaxies from Daddi et al. (2015) and convert this to a molecular gas mass assuming an $\alpha_{\mathrm{CO}}=3.6$ (Daddi et al. 2010), similar to the Galactic value (e.g., Bolatto et al. 2013).

For the galaxies detected in $\mathrm{CO}(2-1)$ up to $z=1.5$, MUSE still covers part of the NUV spectrum (see Fig. 1), where we can use emission line ratios to probe the metallicity. We use the ratio of [O II] $\lambda 3726,3729 /[\mathrm{Ne}$ III] $\lambda 3869$ to infer the gas-phase metallicity in these galaxies, following the calibration from Maiolino et al. (2008), as shown in the left panel of Fig. 3. Overall, we find that the ASPECS galaxies have a (super-)solar metallicity, consistent with what would be expected from the mass-metallicity relation (e.g. Zahid et al. 2014), supporting the use of a Galactic $\alpha_{\mathrm{CO}}$ for these galaxies.

A key question is what kind of galaxies we detect with the flux-limited observations from ASPECS. In Fig. 2, we plot histograms of the stellar masses of the galaxies in which we detect molecular gas. What is immediately evident is that we detect molecular gas in the majority of the most massive galaxies (in terms of their stellar mass). As we move towards lower mass galaxies, the fraction of galaxies decreases, reaching a detection fraction of $\sim 50 \%$ for galaxies with $M_{*} \geqslant 10^{10}\left(10^{10.5}\right) \mathrm{M}_{\odot}$ at $1<z<2(2<z<3)$.

In the right panel of Fig. 3, we expand the one dimensional histograms and show the ASPECS galaxies on the stellar mass - SFR plane. At $1<z<2$, we find that our gasmass selected sample consists mostly of galaxies on the 'galaxy main sequence' at these 

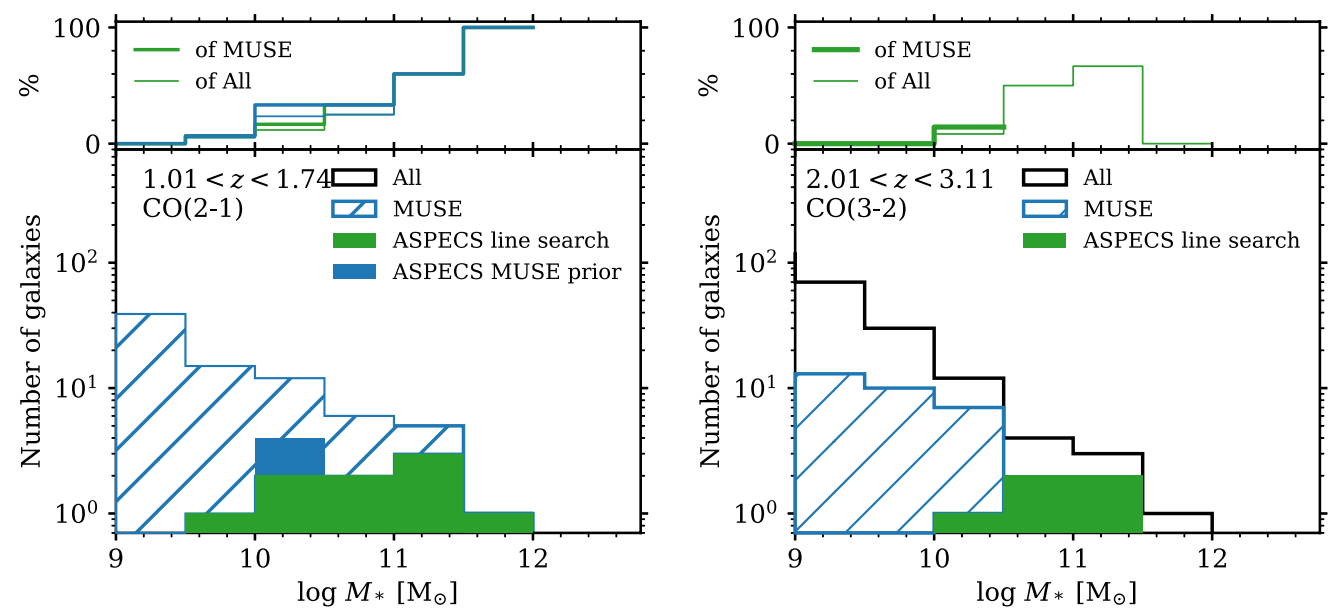

Figure 2. Detection fractions and histograms as a function of the stellar masses $\left(M_{*}\right)$ of the ASPECS galaxies detected in $\mathrm{CO}(2-1)$ (left panel) and $\mathrm{CO}(3-2)$ (right panel). With a purely gas-mass selected sample we detect most of the massive galaxies, while probing down to $M_{*} \approx 10^{9.5} \mathrm{M}_{\odot}$. Figure adapted from Boogaard et al. (2019)
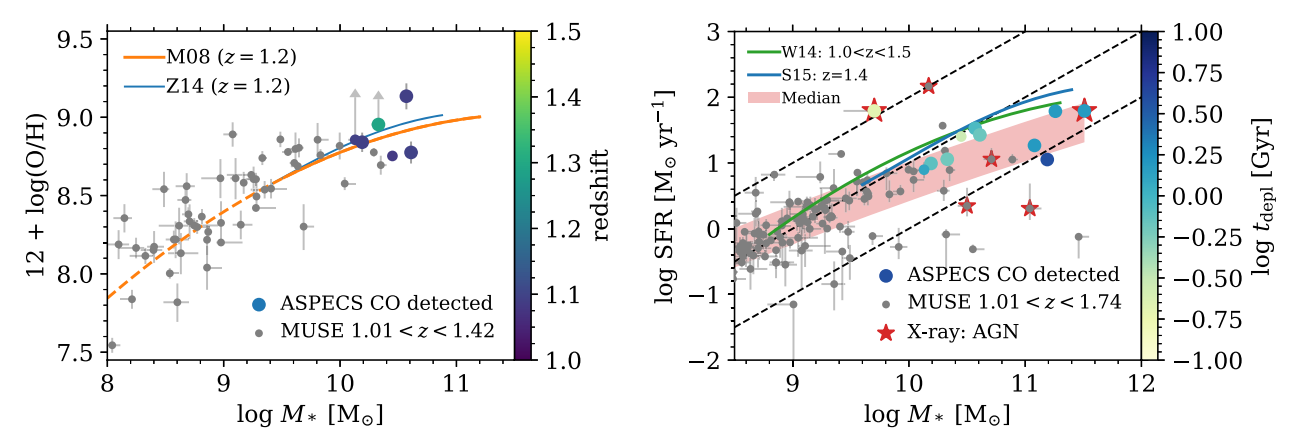

Figure 3. Left panel: Stellar mass - metallicity relation of the ASPECS galaxies detected in $\mathrm{CO}(2-1)$, derived from the $[\mathrm{O} \mathrm{II}] /[\mathrm{Ne} \mathrm{III}]$ ratio (at $z<1.42$ ). The ASPECS galaxies are consistent with a (super-)solar metallicity. Right panel: Stellar mass - star formation rate relation for the ASPECS galaxies detected in CO(2-1). With ASPECS with detect galaxies on, above and below the 'galaxy main sequence' at these redshifts. Figures adapted from Boogaard et al. (2019)

redshifts. Above $M_{*} \approx 10^{10} \mathrm{M}_{\odot}$, we detect almost all of the 'starburst' galaxies, defined as lying $\geqslant 0.3$ dex above the main sequence. Critically, we also detect galaxies that lie below the MS. While these galaxies have SFR that is lower than typical at their stellar mass, they still host a significant gas reservoir. Their detection in the flux-limited survey is important, as these galaxies would typically not be selected in targeted follow-up.

The detections fractions as a function of mass and SFR, together with additional CO lines found using the MUSE redshifts as a prior, suggest that there may be several galaxies for which the $\mathrm{CO}$ emission falls just below the individual detection threshold. The large number of systemic redshifts from MUSE can be leveraged here, in order to recover this signal through stacking. Indeed, preliminary results show that stacking the $\mathrm{CO}(2-1)$ undetected galaxies on the MS at $10<\log M_{*}\left[\mathrm{M}_{\odot}\right]<11$ show a detection of their CO line emission. These and additional results from the stacking will be discussed in Inami et al. (in prep.). 


\section{Outlook: ASPECS and JWST}

The combination of integral field spectroscopy with MUSE in the optical and ALMA in the (sub-)millimeter regime provides a unique tool for studying the physical properties of galaxies across cosmic time. The recently observed band 6 data from ASPECS provide additional constraints on the molecular gas content, through the deep dust continuum map at $1.2 \mathrm{~mm}$ and observations of atomic carbon, as well as on the CO excitation, by constraining higher- $J$ CO lines for all sources.

The anticipated launch of the James Webb Space Telescope will open up a new window on these galaxies. In particular, key rest-frame UV, optical and near-infrared spectroscopy will allow further characterization of the star formation rates, metallicities and ISM conditions in these galaxies. Together with observations of the cold ISM from ALMA, these will provide critical constraints on our theory of star formation at high redshift.

\section{Acknowledgements}

The work presented here is the result of a large collaborative effort and would not have been possible without Fabian Walter, Roberto Decarli, Manuel Aravena, Jorge GonzálezLópez, Chris Carilli, Paul van der Werf, Rychard Bouwens, Roland Bacon, Hanae Inami, and the other members from the ASPECS and MUSE GTO teams. The author would like to thank Paul van der Werf for providing comments on the manuscript.

\section{References}

Aravena, M., Decarli, R., Gónzalez-López, J., et al. 2019, ApJ, 882, 136

Bacon, R., Accardo, M., Adjali, L., et al. 2010, in Proc. SPIE, ed. I. S. McLean, S. K. Ramsay, \& H. Takami, Vol. 7735, 773508

Bacon, R., Conseil, S., Mary, D., et al. 2017, A\&A, 608, A1

Bolatto, A. D., Wolfire, M., \& Leroy, A. K. 2013, ARA\& A, 51, 207

Boogaard, L. A., Brinchmann, J., Bouché, N., et al. 2018, A\&A, 608, A10

Boogaard, L. A., Decarli, R., González-López, J., et al. 2019, ApJ, 882, 140

Brinchmann, J., Charlot, S., White, S. D. M., et al. 2004, MNRAS, 351, 1151

Carilli, C. L., \& Walter, F. 2013, ARAEAA, 51, 1

Daddi, E., Bournaud, F., Walter, F., et al. 2010, ApJ, 713, 686

Daddi, E., Dannerbauer, H., Liu, D., et al. 2015, A\& $A$, 577, A46

Decarli, R., Walter, F., Carilli, C., et al. 2014, ApJ, 782, 78

Decarli, R., Walter, F., Gónzalez-López, J., et al. 2019, ApJ, 882, 138

González-López, J., Decarli, R., Pavesi, R., et al. 2019, ApJ, 882, 139

Inami, H., Bacon, R., Brinchmann, J., et al. 2017, A\&GA, 608, A2

Madau, P., \& Dickinson, M. 2014, ARA $\mathcal{E} A, 52,415$

Maiolino, R., Nagao, T., Grazian, A., et al. 2008, A\&A, 488, 463

Noeske, K. G., Weiner, B. J., Faber, S. M., et al. 2007, ApJ, 660, L43

Pavesi, R., Sharon, C. E., Riechers, D. A., et al. 2018, ApJ, 864, 49

Popping, G., Pillepich, A., Somerville, R. S., et al. 2019, ApJ, 882, 137

Riechers, D. A., Pavesi, R., Sharon, C. E., et al. 2019, ApJ, 872, 7

Schreiber, C., Pannella, M., Elbaz, D., et al. 2015, A\& A, 575, A74

Silverman, J. D., Rujopakarn, W., Daddi, E., et al. 2018, ApJ, 867, 92

Tacconi, L. J., Genzel, R., Saintonge, A., et al. 2018, ApJ, 853, 179

Walter, F., Decarli, R., Sargent, M., et al. 2014, ApJ, 782, 79

Walter, F., Decarli, R., Aravena, M., et al. 2016, ApJ, 833, 67

Whitaker, K. E., Franx, M., Leja, J., et al. 2014, ApJ, 795, 104

Zahid, H. J., Dima, G. I., Kudritzki, R.-P., et al. 2014, ApJ, 791, 130 